\title{
التنوع الثقافي ودوره في إحداث التنمية المستدامة وتقييمه
}

\section{CULTURAL DIVERSITY AND ITS ROLE IN THE CREATION AND}

\author{
EVALUATION OF SUSTAINABLE DEVELOPMENT \\ تاريخ الاستلام :2019/051/23؛ تاريخ القبول : 2019/05/30
}

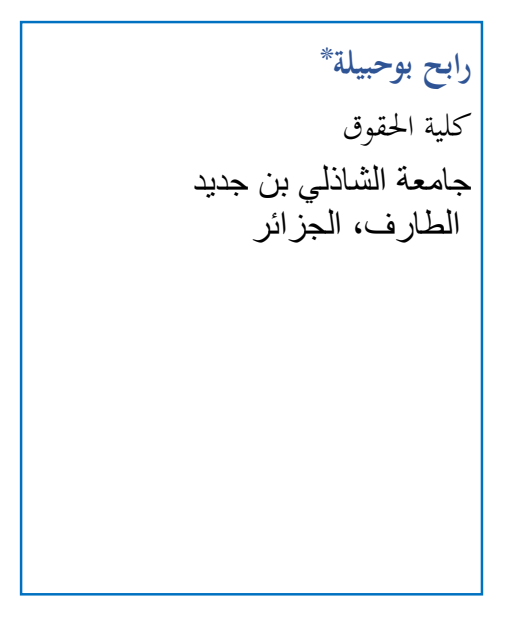

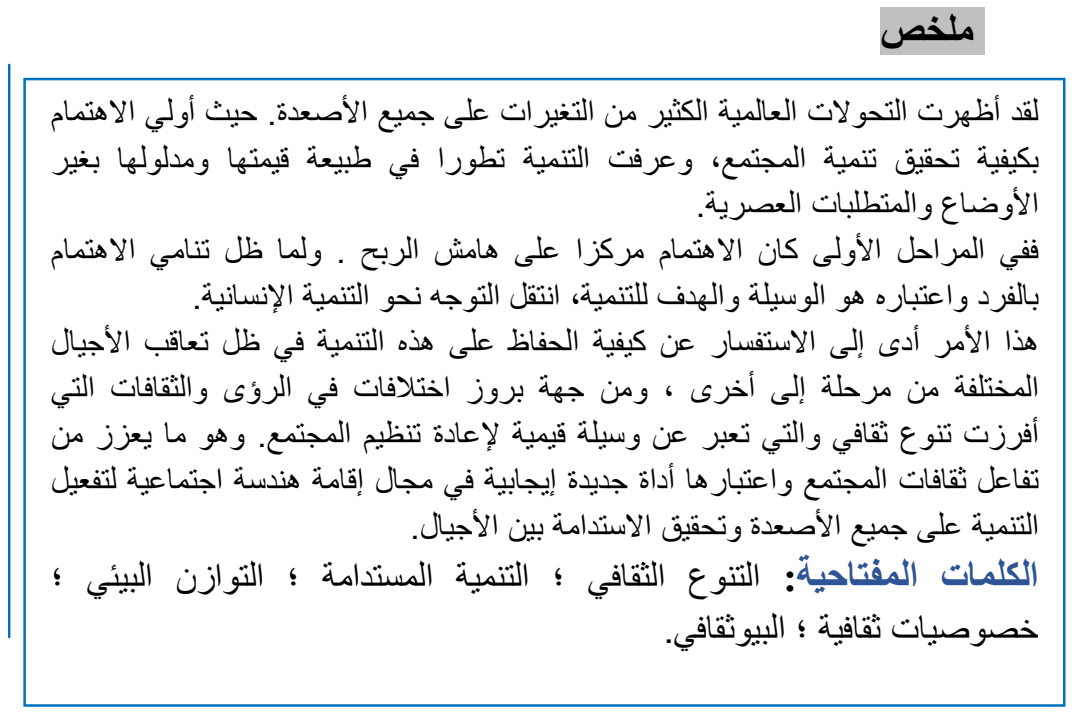

\section{Abstract}

The global transformation produced many changes at all levels where attention was how to achieve the development of society, as it is known development in the evolution of the nature of the value and significance without modern conditions and requirements, in the beginning the focus was on profit margins while in light of the growing concern for individual and regarded as a means and gool of development is the trends humanitarian development. And this has lad to ask how to keep this development under generational succession. Which very from one stage to another, hand differences displayed on the vision and cultures that produced great cultural diversity which reflects the ad valoren tool for the reorganization of society and This Is chat enhances the interaction of the cultures of the community and consider the mechanism. Of a new positive in the Field of social engineering established to activate the development at all levels until you can achieve sustainability between the generations. But this paices the problem of our study are as follows.

Keywords: Cultural diversity , sustainable development, environmental balance, cultural, biocultural specificities

\section{Résumé}

La transformation globale a provoqué de nombreux changements à tous les niveaux où l'attention était de savoir comment réaliser le développement de la société, car il est connu que l'évolution dans la nature de la valeur et de la singularité sans conditions et exigences modernes, à l'origine, l'accent était mis sur le profit Le développement humain est la tendance actuelle du développement humain, à la lumière de la préoccupation croissante de chacun et considéré comme un moyen de développement. C'est pourquoi nous devons nous demander comment maintenir ce développement dans la succession des générations. D'un stade à l'autre, les différences entre les mains affichées sur la vision et les cultures ont donné lieu à une grande diversité culturelle qui reflète l'outil ad valorentool pour la réorganisation de la société et This is chat renforce l'interaction des cultures de la communauté et considère le un nouveau point positif dans le domaine de l'ingénierie sociale mis en place pour activer le développement à tous les niveaux jusqu'à atteindre la durabilité entre les générations. Mais ce problème le problème de notre étude sont aussi évidents.

Mots clés: Diversité culturelle, développement durable , équilibre environnemental, spécificités culturelles , bioculturelles

*Corresponding author, e-mail: bouhebila2018rabah@gmail.com 


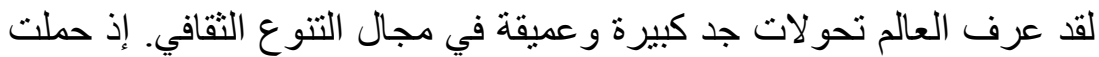

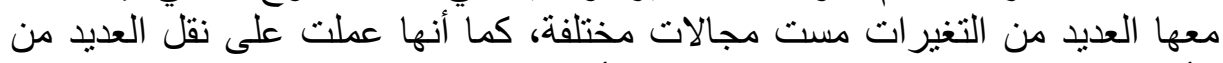

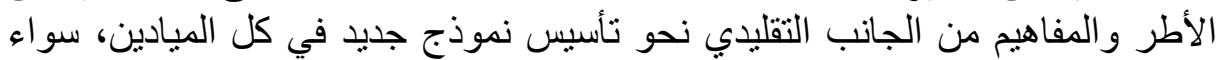
من الناحية الاقتصادية أو السياسية أو الاجتماعية.

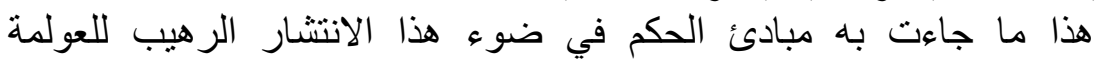

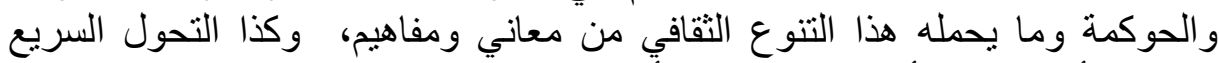

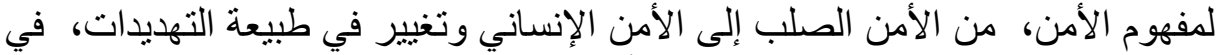

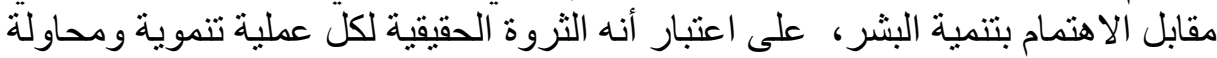

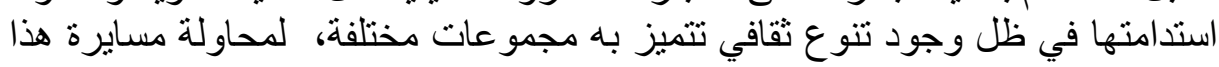
التنو ع لخلق تنمية مجتمعية متكاملة. لذا يمكن القول أن التتوع التقافي هو ذللك الاختلاف الثقافي، وهو حقيقة واقعية

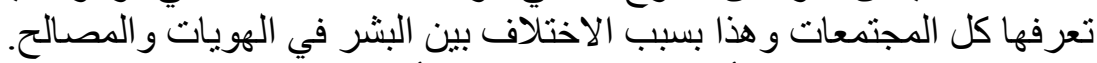

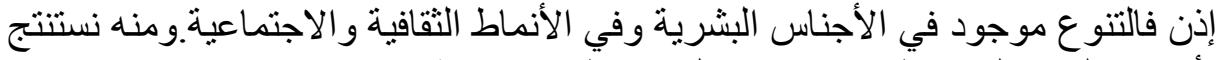

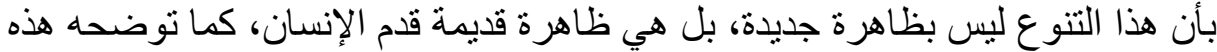

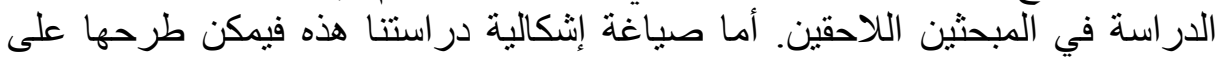

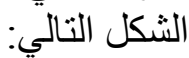

إلى أي مدى يمكن تحقيق التنمية في بعدها المستدام في ظل التتوع الثقافي المجتمعي

المبحث الأول: التنوع الثقافي لإحداث التنمية المستدامة

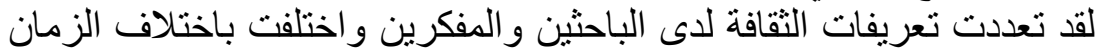

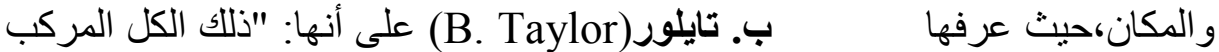

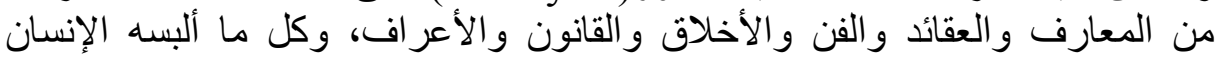

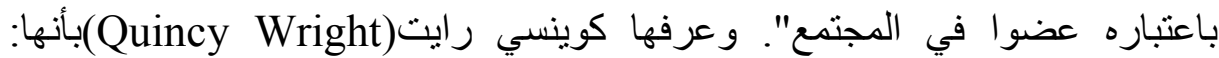

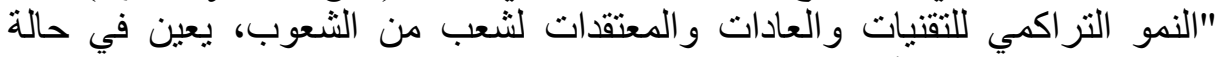

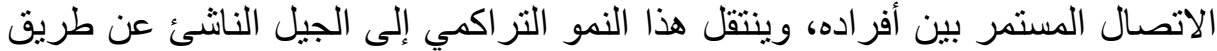

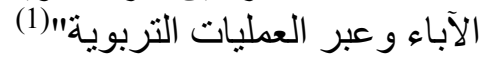

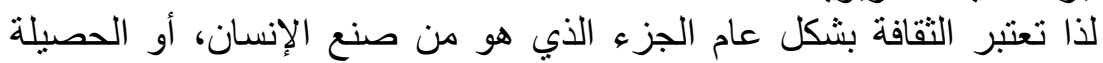

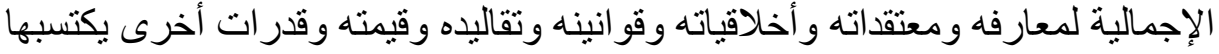
الإنسان كعضو في المجتمع.

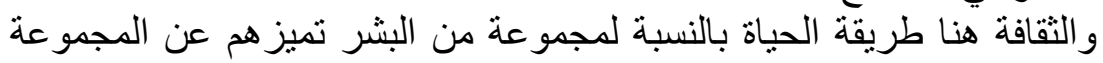

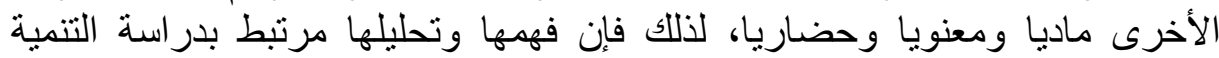

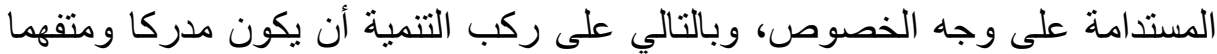

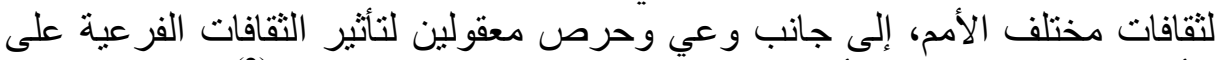

الأنماط السلوكية، ومن ثم أثر ها على استر اتيجيات التنمية المستدامة.

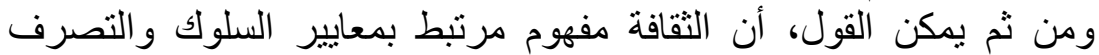

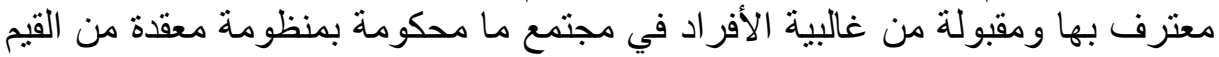

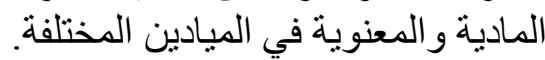
المطلب الأول: مفهوم التنوع الثيادين الثقافي

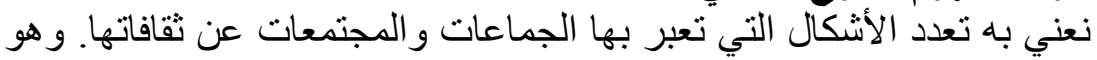
يعكس الاختلافات الناتجة عن الخيار ات الفردية. فالتنوع الثقافي يمثل فكرة التعايش بين 
أكثر من مظهر ثقافي داخل نفس الوسط المجتمعي، وعندما يكون لاى المجتمعات

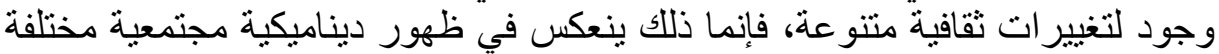

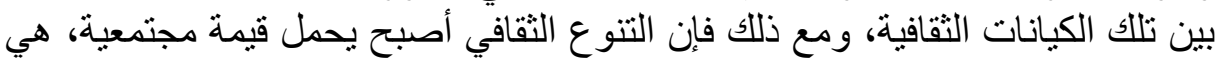
قيمة توجيهية من شأنها أن توفر نو عا من خارطة الطريق لتان للتنظيم الاجتماعي و التقافي

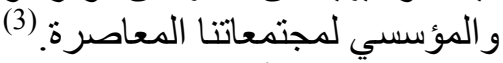

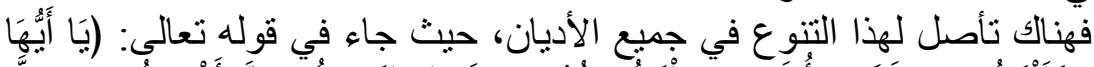

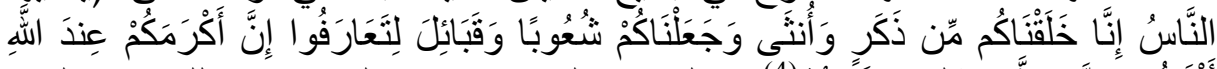

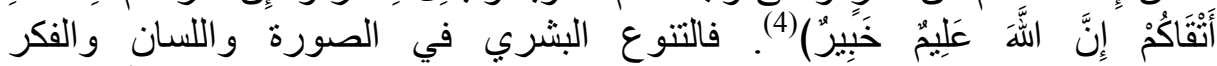
و العمل.فالتنوع الثقافي هو ميراث للإنسانية جمعاء وينبغي الاعتر اف به والته والتأكيد عليه

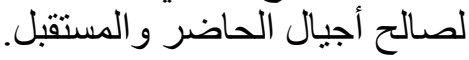
و هنالك العديد من المصطلحات المشابهة لهذا التنوع، منها: التعددية التقافية،

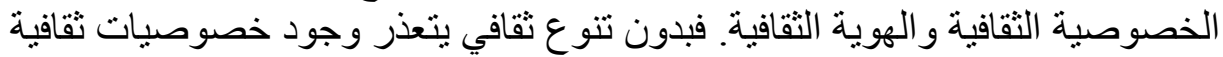

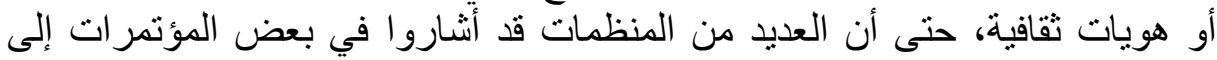

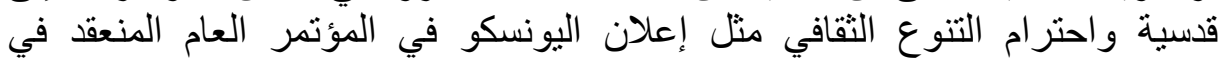

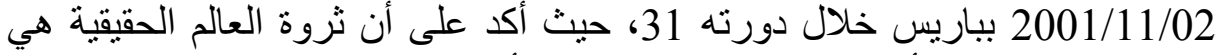

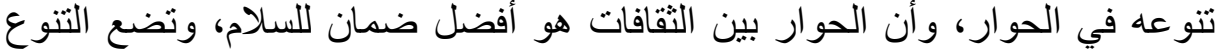

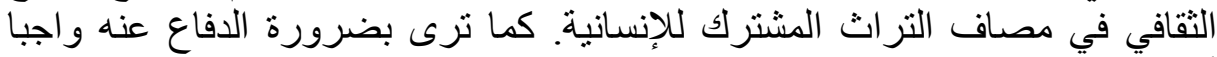

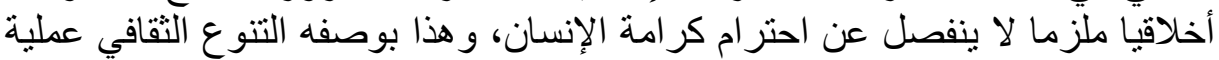

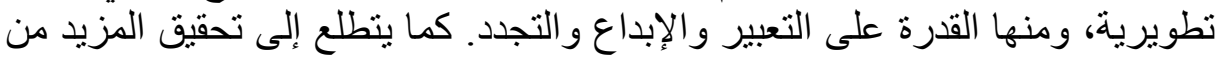

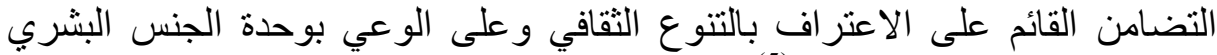

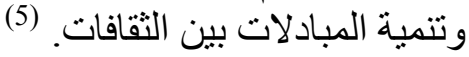
و على هذا الأساس يمكن تأكيد القول على أنى أن التنوع الثقافي يعد مصدر التهات التقديم

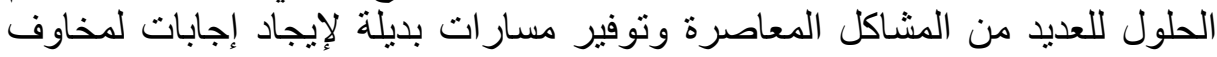

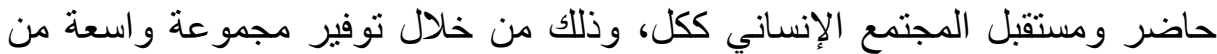

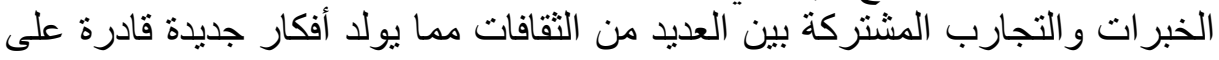

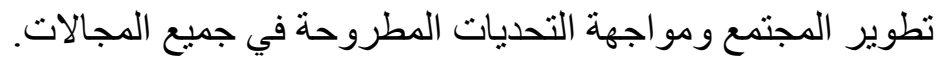

المطلب الثاني: التنمية المستدامة (Sustainable development)

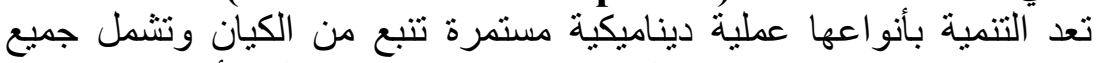

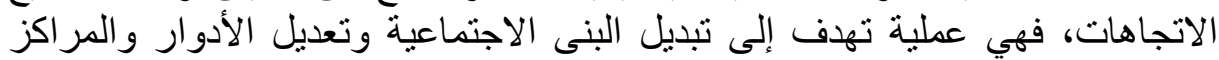

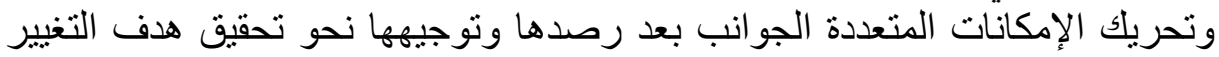

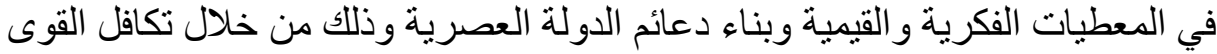

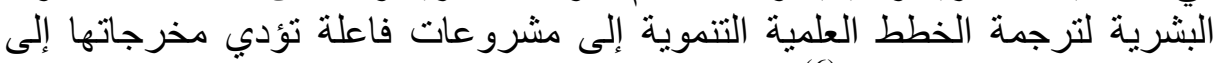

إحداث التغير ات المطلوبة.

لقد استخدم (Sustainability)

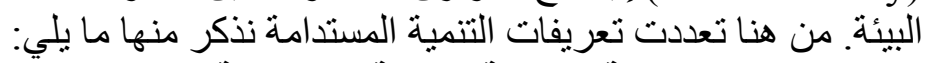

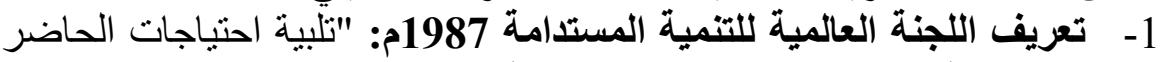
دون أن تؤدي إلى تدمير قدرة الأجيال المقبلة على تلبية المبلة احتياجاتها 2- تعريف مؤتمر الأمم المتحدة للبيئة والتنمية ـريو دي جانيرو-1992م:

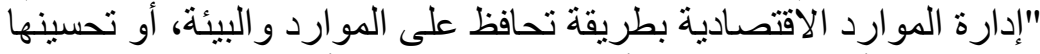

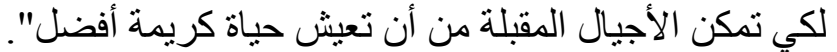

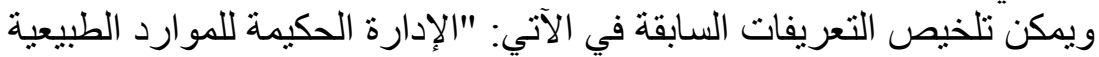

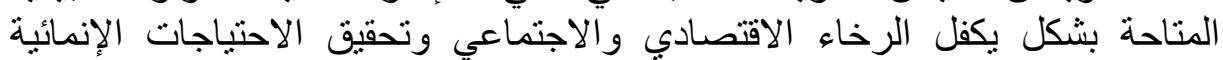


ولعل مقو لة التنمية: " أمست اليوم محور ا مشتركا لمعظم العلوم الإنسانية وتطبيقاتها".

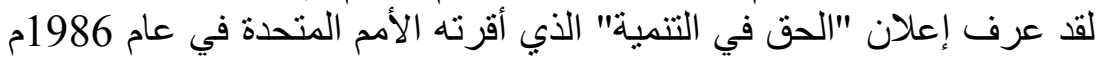

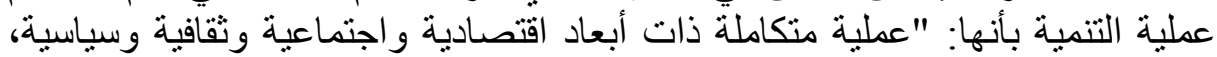

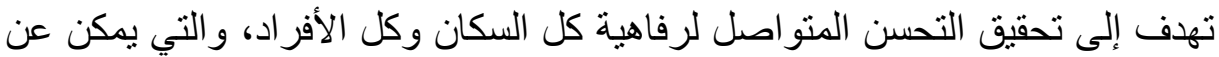
طريقها ضمان حقوق الإنسان وحرياته الأساسية". فالتنمية المستدامة تهدف الإنى التوانه التق و التكامل بين البيئة و التتمية من خلال

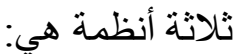
1-نظام حيوي للموارد: ويعني القدرة على التكيف مع المتغيرات الإنتاجية البيولوجية

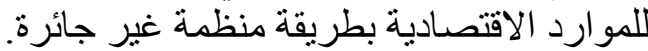

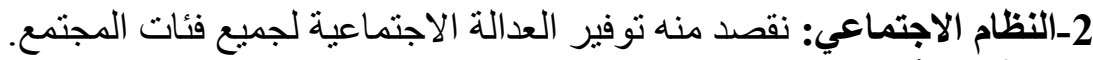

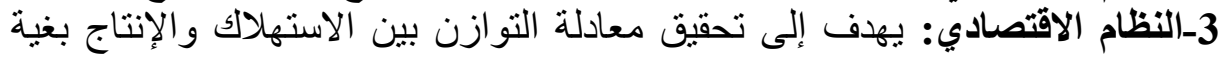

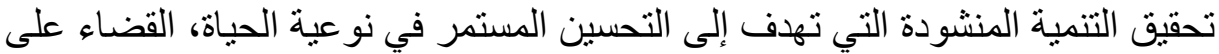

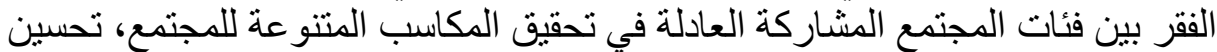

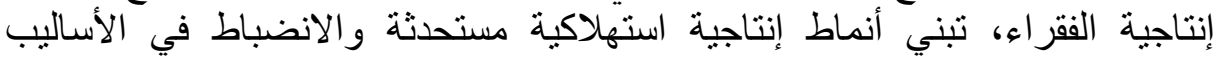

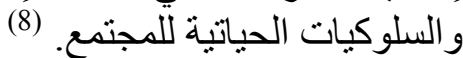
ووفقا لكل التعاريف المقدمة للتنمية المستدامة، نجد أنها مستمدة من مبادئها الثناث و هي: التقدم الاقتصادي، العدالة الاجتماعية وحماية البيئة.

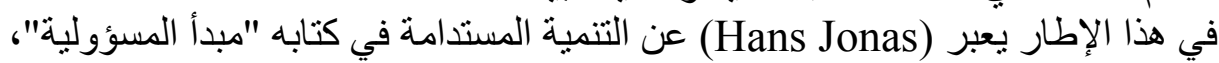
حيث يدمج المفاهيم الثناث للتنمية من خلال الثنكل التوضيحي التالي:

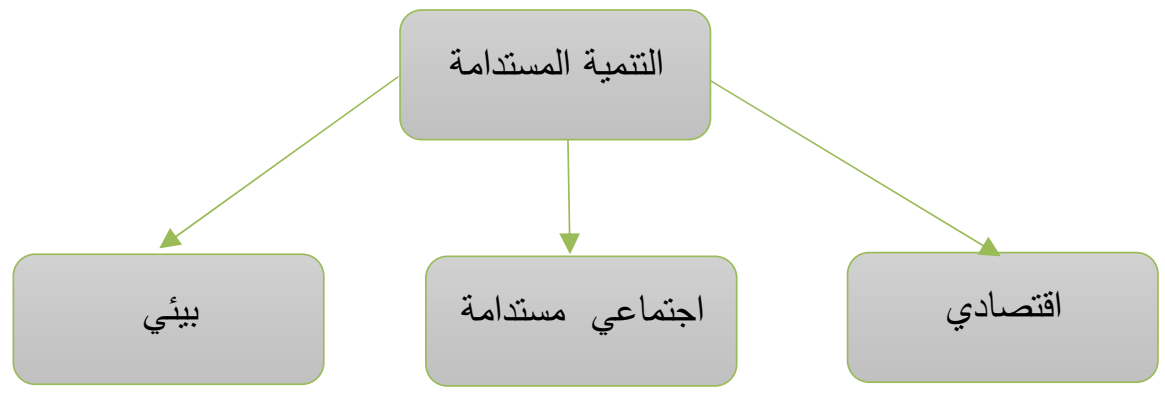

مخطط يبين الأسس الثلاث التي ترتكز عليها التنمية المستدامة، حسب قمة الأرض بجو هانسبورغ لعام 1992، و هي: "الركيزة الاقتصادية و الاجتماعية و البيئية".

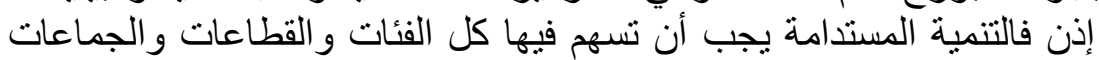

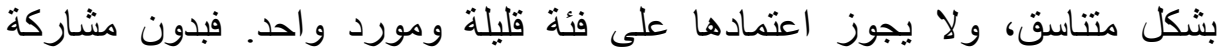

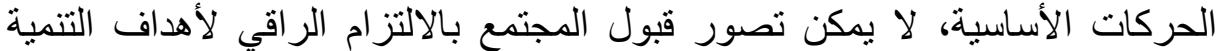

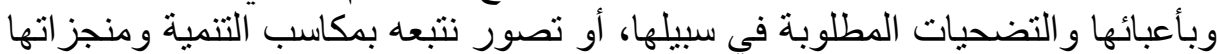

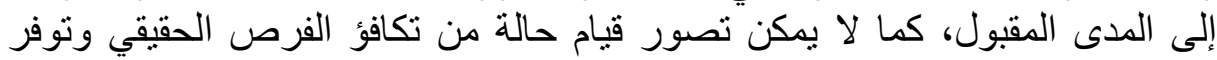

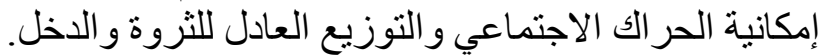

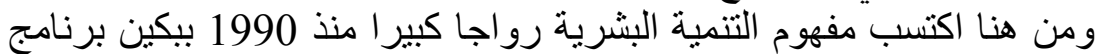

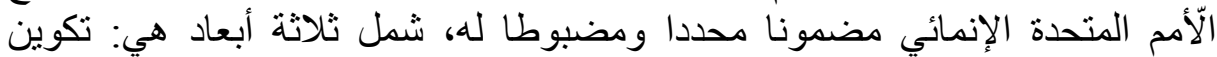

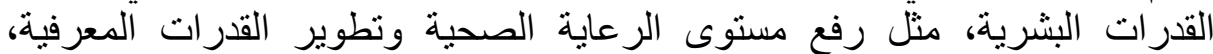

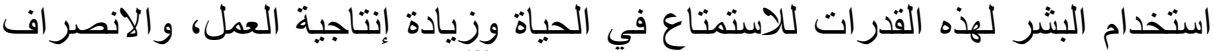

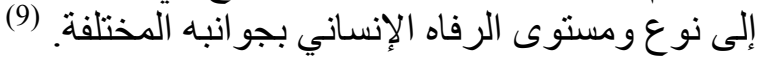


المبحث الثاني: العلاقة التفاعلية بين التنوع الثقافي والتنمية المستدامة

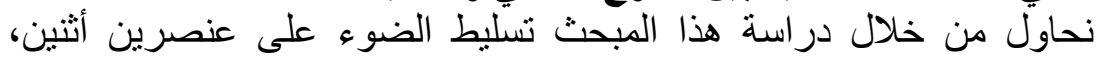

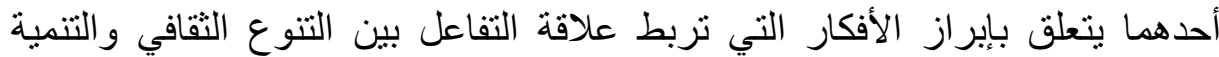

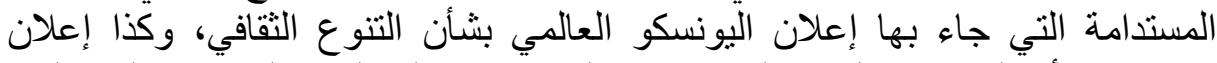

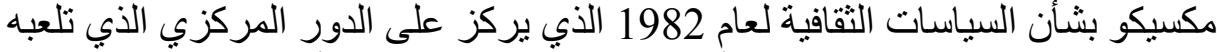

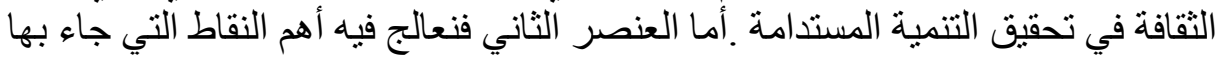

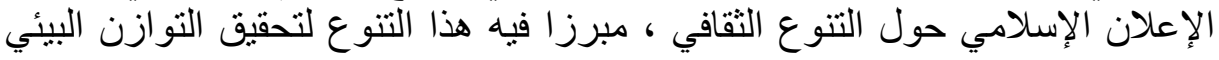

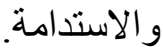

\section{المطلب الأول: التنوع الثقافي كبعد لتحقيق التنمية المستدامة}

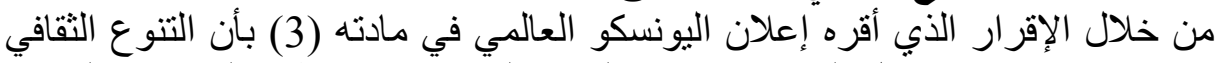

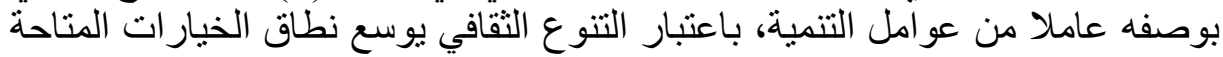

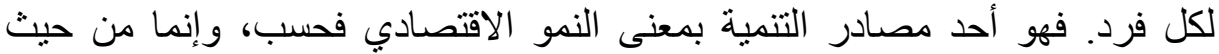

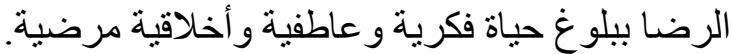

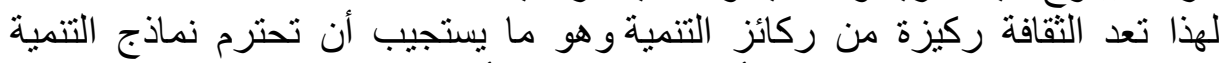

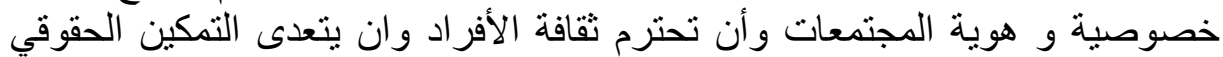

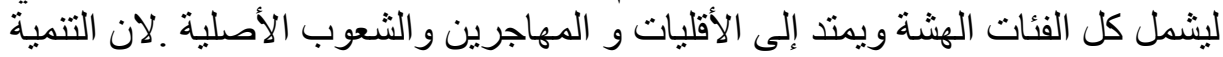

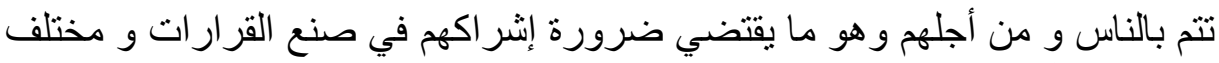

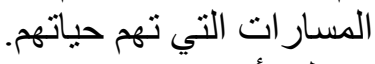
لقد أكد إعلان ماكسيكو بشأن السياسات الثقافية لعام 1982 على الدور المركزي

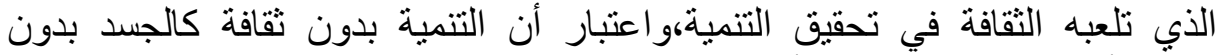

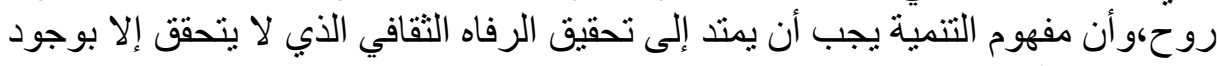
بر امج تتموية تضمن إستمر اريته و ديمو ميتها.

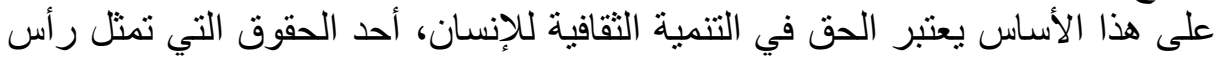

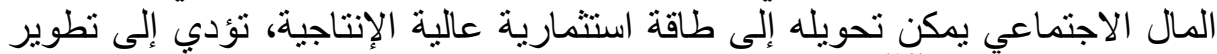

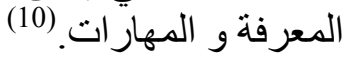

كما انه لا يمكن الحديث عن الاستقر ار السلمي للحياة، إلا في ظل إقرار الإ حقيقي بحق كل

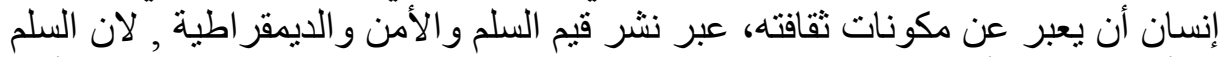

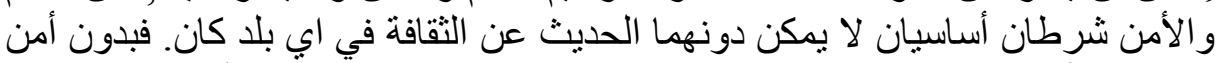

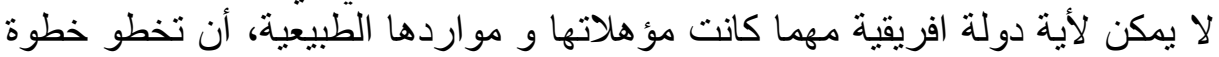

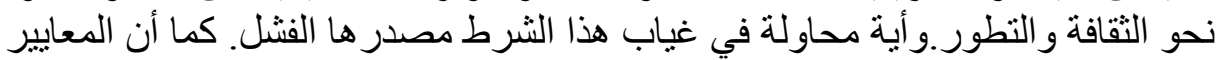
العالمية للديمقراطية وحقوق الإنسان تطلب منا المزيد من لعمل الجاد قصد تصائ تفعيل

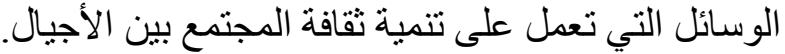

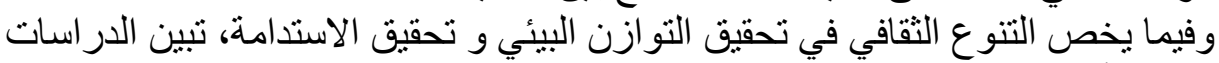

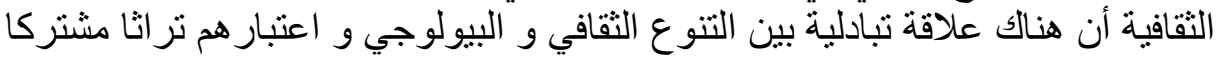

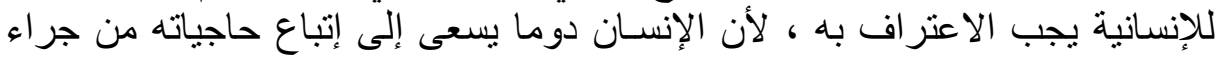

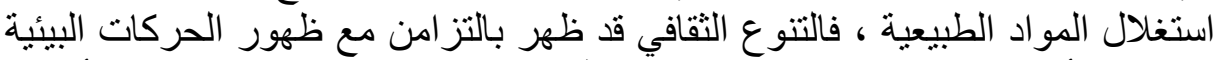

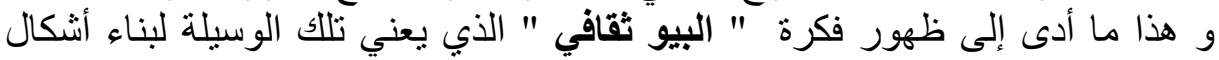
تحسيسية تربط الإنسان بييئته الطبيعية و هو نتيجة حقيق التيقية لعلاقة الترابط الوثيق بين

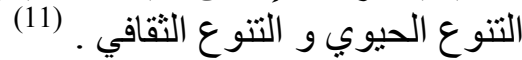
فالتنمية في تكامل أبعادهاً الاقتصادية و الفافئو الاجتماعية و البيئية تتطلب إطار أخلاقي

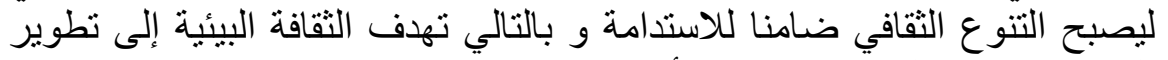

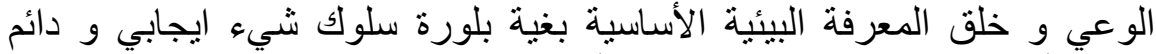

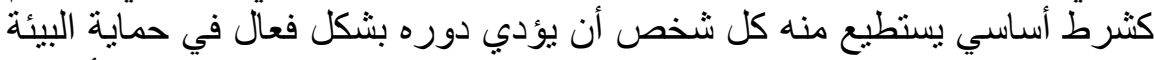

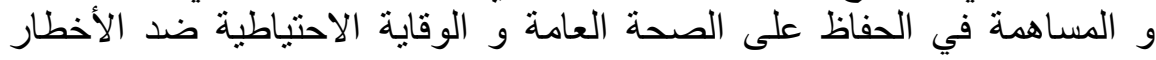


البيئية طبقا لوجهات النظر الايكولوجية و الاقتصادية و الاجتماعية لضمان مستقبل

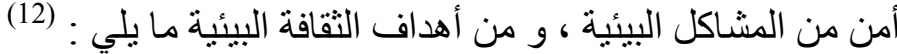

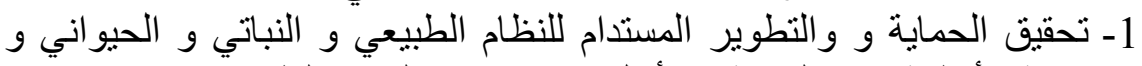

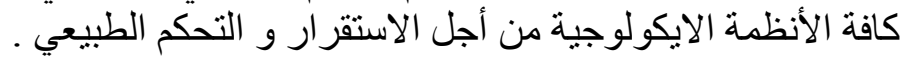

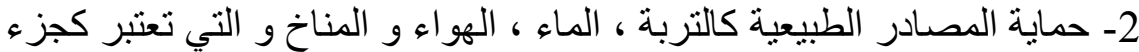

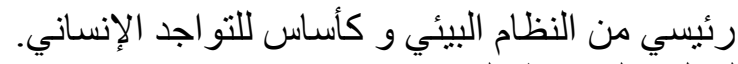

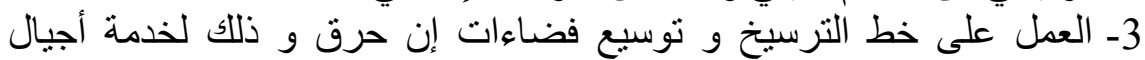

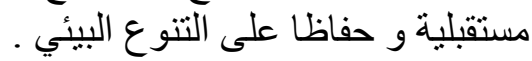

4- الوقاية الاحتياطية من المشاكل البيئية المستقبلية و الممكن تداركها.

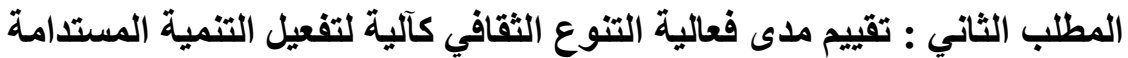

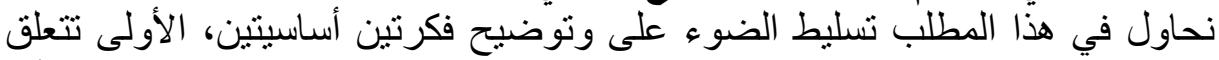
بالطرح الإيجابي الذي يؤكد على الدور الفعال للتنوع الثقافي في تحقيق الاستدامة، أما

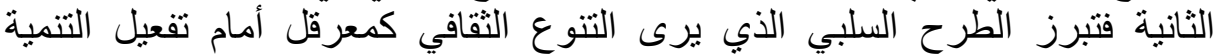

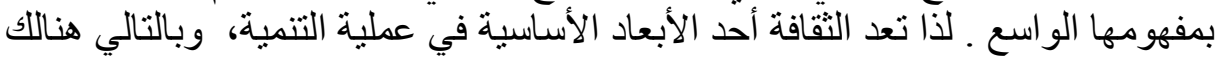

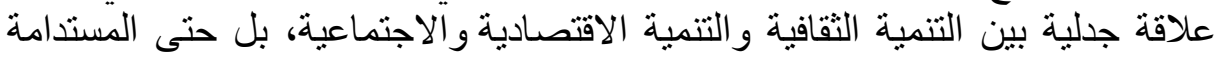

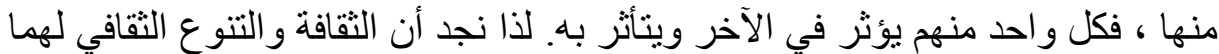
دورا إيجابيا في تفعيل سبل التنمية منها: أــ يعد التنوع الثقافي مصدر الإثر اء الثقافة البشرية التي يثير بها التو اصل مع الثقافات الفردية المغايرة لها. ب- ينطوي التنوع على شيء من القيم الجمالية في خلق عالم متنوع و غني في الرأي

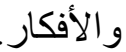
ج- يخلق التنوع الثقافي مناخا تشترك فيه الثقافات المختلفة بحوار يعود بالنفع عليها

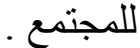
د- التنوع الثقافي أساس حماية حقوق الإنسان وتطبيق الديمقر اطية بطريقة صحيحة

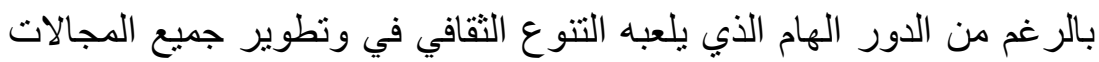

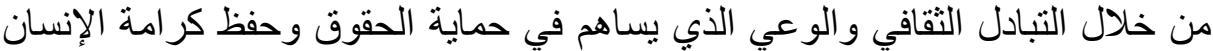

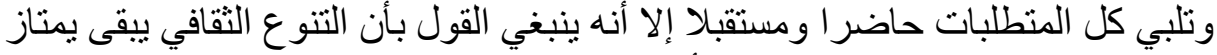

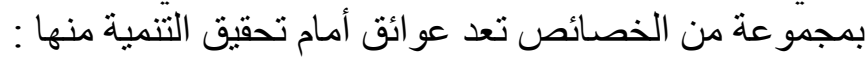

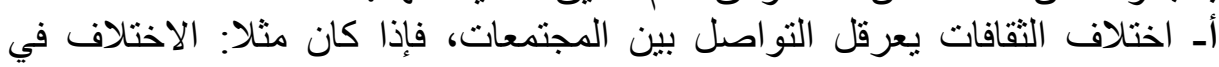
العنصر اللغوي بين الثقافات، كثير ا ما يكون مصدر الصعوبة التئ في التواصل الصن.

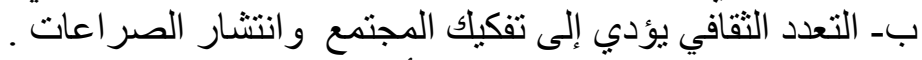

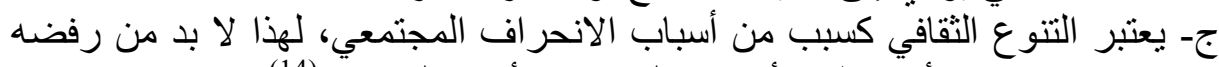

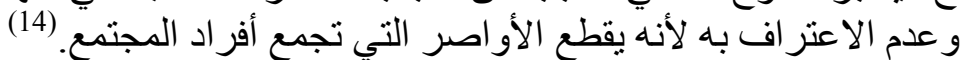

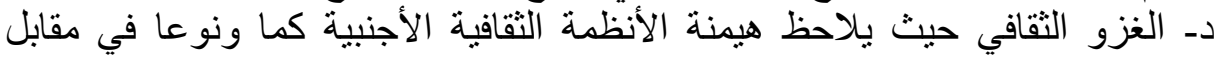

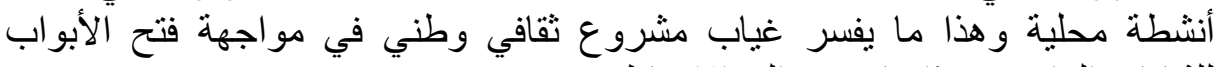

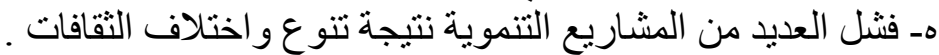


مما تقدم يمكن أن نخلص بان ظاهرة التنوع الثقافي ليست بظاهرة جديدة، و إنما

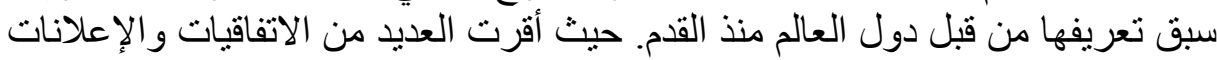

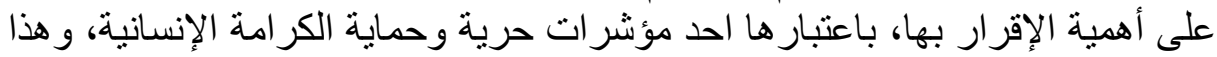

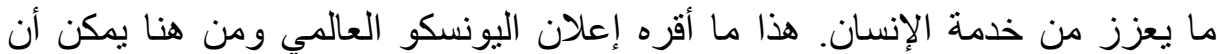
نضع النتائج التالية: من فئة 1- ظاهرة التنوع الثقافي ليست ظاهرة جديدة وإنما عرفتها العديد من دول

$$
\text { العالم منذ القدم. }
$$

2- يعتبر التنوع الثقافي أحد مؤشرات حرية وحماية الكرامة الإنسانية، وهذا

$$
\text { ما يعزز من خدمة الإنسان. }
$$

3- التنوع الثقافي يوسع نطاق الخيار ات المتاحة لكل فرد، وهو الاند احد مصادر

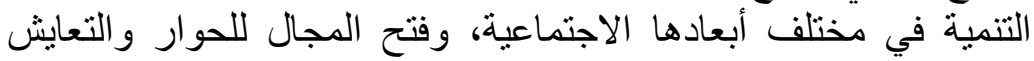

$$
\text { السلمي. }
$$

4- التتوع الثقافي يفتح الأبواب في المجال الاقتصادي للتعامل مع الأسواق الخارجية ورفع مسنوى الإنتاج.

5- التنوع الثقافي في المجال البيئي يمنح الفرصة لإني للاستغلال الأمثل لثروات

الطبيعة وتوفير الحاجات لكل الأجيال حاضر الومستقبلا.

(1)- نصيرة صـالحي، التنوع الثقافي كآلية جديدة لتفعيل مسار التنمية المستدامة، أعمال

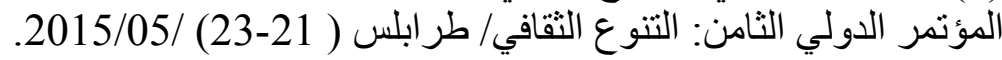

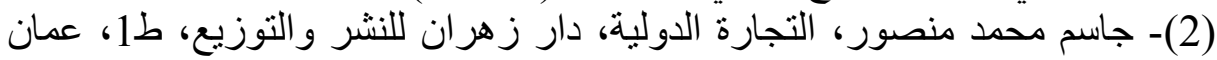

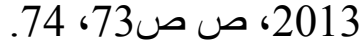
(3)- سناء ضيف، التنوع الثقافي من منظور الأمن المجتمعي، مذكرة ماجستير جامعة

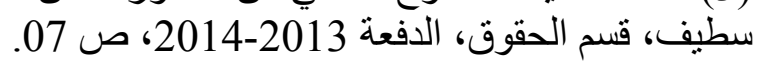

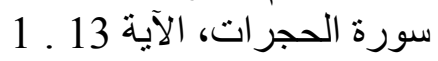

(4)- إعلان اليونسكو العالمي بشأن التنوع الثقافي، باريس 2001، المادة (1).

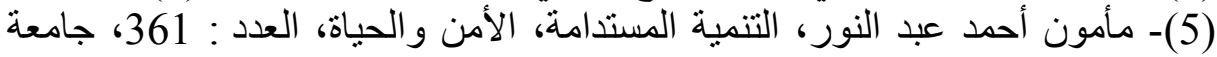

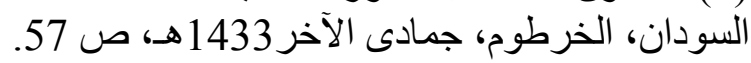

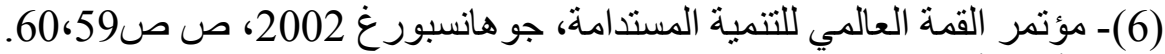

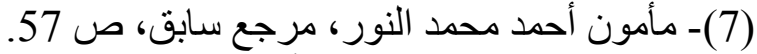

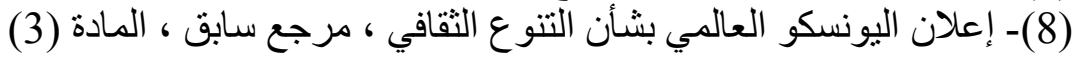

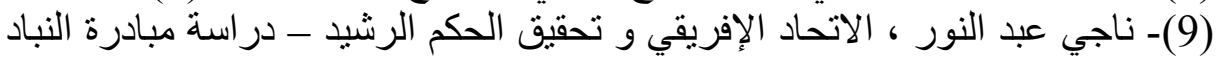

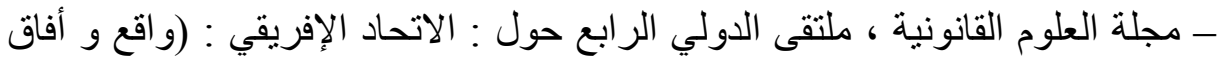

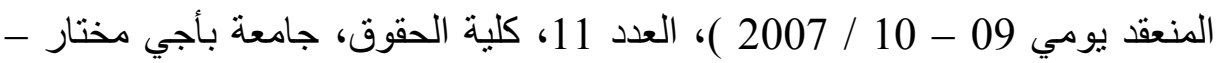

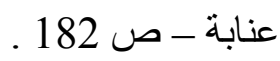

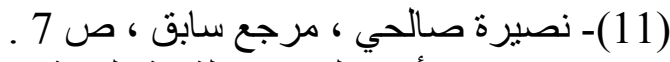

(12)- عزوي ، أحمد لعمي ، الثقافة البيئية بعد إستراتيجي لحماية البيئة ، تم تصفح

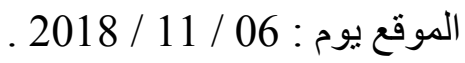

(13)- ييشرة محمد أمين، آليات الحكم الرشيد في إدارة التتوع الثقافي - در اسة تحليلية

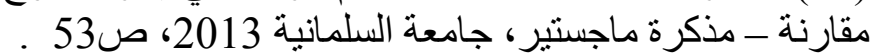

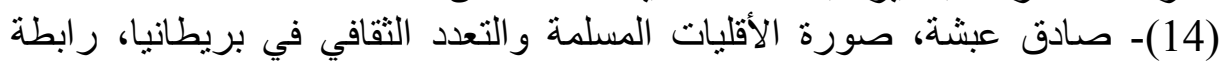
العالم الإسلامي، تونس 2012-2013 